\title{
Evidence for the efficacy of the MORI technique: Viewers do not notice or implicitly remember details from the alternate movie version
}

\author{
Lauren French, Matthew P. Gerrie, and Maryanne Garry \\ Victoria University of Wellington, Wellington, New Zealand \\ AND \\ KAZUO MorI \\ Tokyo University of Agriculture and Technology, Tokyo, Japan
}

\begin{abstract}
The MORI technique provides a unique way to research social influences on memory. The technique allows people to watch different movies on the same screen at the same time without realizing that each of them sees something different. As a result, researchers can create a situation in which people feel as though they share an experience, but systematic differences are introduced into their memories, and the effect of those differences can be tracked through a discussion. Despite its methodological advances, the MORI technique has been met with criticism, mostly because reviewers are worried that the MORI technique might not completely block the alternate movie version from view, leading people in these studies to see their partner's version of the movie as well as their own. We addressed these concerns in two experiments. We found no evidence that subjects noticed the alternate movie version while watching a movie via the MORI technique (Experiment 1) and no evidence that subjects remembered details from the alternate movie version (Experiment 2). Taken together, the results provide support for the MORI technique as a valuable research tool.
\end{abstract}

Mori and colleagues developed a technique, called the MORI technique, to present different viewers with different images on the same screen at the same time (Mori, 2003, 2007). The MORI technique uses the principles of light polarization. Specifically, two projectors project two different movies so that the images overlap on a screen; filters polarize the image from one projector vertically and the image from the other projector horizontally. People wear polarizing glasses that allow them to see one version of the movie but block the other version from view. In short, two-or more - people can sit side by side and, ostensibly, watch the same movie on the same screen but actually see different things (see Figure 1).

The MORI technique provides a unique way to research social influences on memory. We know that people often remember the same events in different ways (Cuc, Ozuru, Manier, \& Hirst, 2006; French, Sutherland, \& Garry, 2006; Ikier, Tekcan, Gulgoz, \& Kuntay, 2003; Loftus, Levidow, \& Duensing, 1992; Sheen, Kemp, \& Rubin, 2001); consequently, when people discuss the past, they may gain access to new information. Although hundreds of studies have demonstrated that postevent suggestion can change people's memories for witnessed events, it is more challenging to demonstrate the effects of discussion on those memories. Researchers must overcome three hurdles: First, they must know what happened during a target event; second, they must introduce systematic discrepancies into the discussion; third, they must assess the effects of those conflicts on people's memories.

Earlier research examining the effects of discussion on memory has been limited in its ability to overcome the last two of these hurdles, relying instead on two other approaches. One approach was to pair a subject with a confederate to examine how the confederate's false responses would affect the subject's responses (see, e.g., Gabbert, Memon, Allan, \& Wright, 2004; Kassin \& Kiechel, 1996; Meade \& Roediger, 2002; Paterson \& Kemp, 2006; Roediger, Meade, \& Bergman, 2001; Schneider \& Watkins, 1996; Shaw, Garven, \& Wood, 1997; Wright, Mathews, \& Skagerberg, 2005). In the second approach, pairs of subjects are separated (in space or time) while each of them views a different event (or set of materials); later, the subjects come together to discuss what they are led to believe was the same experience (see, e.g., Cuc et al., 2006; Gabbert, Memon, \& Wright, 2006, 2007; Wright et al., 2005; Wright, Self, \& Justice, 2000).

But neither approach fully captures the subjective experience of sharing an event with another person and discussing it together. For one thing, confederates may behave fundamentally differently from people in natural discussions. Confederates are told to convey the misleading information confidently and to avoid commenting on

M. Garry, maryanne.garry@vuw.ac.nz 


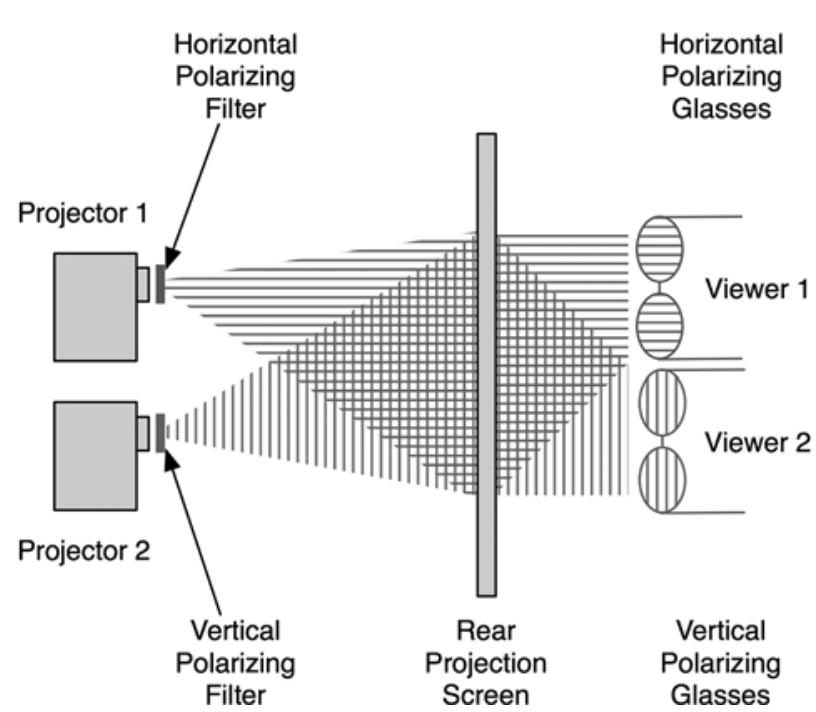

Figure 1. Illustration of equipment setup for MORI technique. Two projectors project two movies onto the screen. One movie is polarized horizontally, and the other is polarized vertically. Viewers wear polarization glasses allowing them to see one movie while blocking the other.

anything that the subject says during discussion (see, e.g., Paterson \& Kemp, 2006). This kind of dialogue may break normal conversational rules, constrain the social dynamics or sociolinguistic factors that contribute to memory conformity, or simply seem strange (Cuc et al., 2006). In addition, experiencing the same event (or what people believe is the same event) as another person while being separated from that person is, on the face of it, not the same as genuinely experiencing an event together.

The MORI technique can overcome both of these shortcomings. It allows people to share an experience: They sit side by side and, ostensibly, watch the same movie on the same screen at the same time. It also allows researchers to introduce systematic discrepancies into the discussion and then assess the effect of those discrepancies on people's memories. We have used the MORI technique several times to examine various social influences on memory (French, Garry, \& Mori, 2008, 2009; Garry, French, Kinzett, \& Mori, 2008; Hirokawa, Matsuno, Mori, \& Ukita, 2006; Kanematsu, Mori, \& Mori, 2003). In most of our studies, the same basic three-stage procedure has been used. First, pairs of people each unwittingly watched slightly different versions of a movie. The movie versions were filmed once and then digitally altered (see, e.g., Garry et al., 2008). As a result, the two movie versions were identical - and overlapped at all points - except during the presentation of eight critical differences. After watching the movie, the pairs answered questions about it together; some questions guided them to discuss details for which they saw contradictory information. Finally, the subjects completed a memory test individually to determine what each person really remembered about the event. Our typical results have suggested that discussion corrupts people's memories: People report seeing things that their partner only told them about during the discussion.

Despite its methodological advances, the MORI technique has been met with criticism, usually during peer review. Reviewers have worried that the technique might not have completely blocked the alternate movie version from view. For instance, if subjects tilted their heads during the movie, the angle of polarization would have changed, allowing them to see aspects of both movies, and thus the alternate movie might not have been completely blocked from view. If the subjects did see both versions of the movie, the details their discussion partner mentioned would not have been new; instead, those discussed details would have been genuinely present in the movie. Such a possibility raises an alternative and unexciting explanation for the typical pattern we and others have found: People reported that they saw misleading details because they really did see them, not because their discussion partner fed them incorrect information.

We find this alternative explanation weak for at least four reasons. First, remember that the movie versions overlap at all points except during presentation of the critical material. If the two images match perfectly and play in sync, even observers wearing no polarizing glasses will see what appears to be only one movie for the majority of the time. For instance, the movies used in these two experiments were identical for 6 min $1 \mathrm{sec}$ out of a total movie duration of $6 \mathrm{~min} 34 \mathrm{sec}$, or $92 \%$ of the time. Thus, even if the subjects did tilt their heads, it is very unlikely that they would do so at the precise moment when two conflicting images appeared on the screen. Second, if subjects do coincidentally tilt their heads during presentation of a critical item, they will not simply see both versions of a detail. More specifically, because the two movie images are overlapped directly over the top of one another, the nonmatching aspects will look like a fuzzy mess rather than two clearly defined versions of a detail. Third, if subjects see two contradictory details-for example, the main character stealing a wallet and putting it back - they should report seeing both when trying to answer the relevant discussion questions. But people do not mention anything about seeing the other movie, nor do they mention anything about seeing different versions of the critical details. In fact, subjects often seem surprised when their partners recall contradictory details during the discussion; if these subjects had also seen the contradictory details during the movie, they should not be surprised by their partner's responses. Finally, if subjects do notice two versions of a critical detail, they should become suspicious about the study goals - perhaps even realizing they were supposed to see something different from what their discussion partner saw. If subjects become suspicious about the study goals, it should lead them to judge their partners as unreliable sources of information and increase their source monitoring; all of these behaviors would reduce the effects of misleading suggestion (Dodd \& Bradshaw, 1980; Echterhoff, Hirst, \& Hussy, 2005; Johnson, Hashtroudi, \& Lindsay, 1993; Lindsay, 2008; Mitchell \& Johnson, 2000; Tousignant, Hall, \& Loftus, 1986). 
But despite the logic of our counterarguments, we admit that there are no empirical data that address these possibilities. For example, it remains a possibility that subjects have noticed the second movie version but, for some reason, have not said anything about it. We addressed these concerns by examining whether subjects would notice the alternate movie version while watching an event presented with the MORI technique (Experiment 1), and whether subjects would remember details from the alternate movie version (Experiment 2).

\section{EXPERIMENT 1}

The purpose of Experiment 1 was to determine whether people would notice the alternate movie version. One problem with simply asking people whether they notice the alternate movie version is that they might report seeing the alternate version to save face, rather than because they actually did notice it. We attempted to limit these kinds of response biases - where the questions demand a certain answer-in several ways (Orne, 1962). First, instead of asking subjects outright if they saw the alternate movie, we asked them to respond to a series of increasingly suggestive questions. Second, to gather a baseline measure of how often subjects would respond "yes" to the critical questions, we used the MORI technique itself as a between-subjects factor. That is, for half of our pairs we used the standard MORI technique: Both versions of the movie were played overlapping on the same screen at the same time, but each person wore polarizing glasses that allowed him or her to see only one version. For the remaining pairs, we used a sham MORI technique where there was no overlapping image: Only one version of the movie played, and both people wore the same polarizing glasses. If people in this condition reported seeing the alternate movie version, it would suggest that they did so due to the demand characteristics of the questions. Finally, we used Laney et al.'s (2008) red herring technique. In the red herring technique, researchers convey a fake cover story about the purpose of the study. Then, to stop the research-savvy subjects from deducing the true purpose of the study on the basis of the questions they encounter, the critical questions are embedded among other questions that hint at another, fake purpose. We used the same cover story as Garry et al. (2008) and French et al. (2008) - that we were interested in people's sensory impressions at different levels of visual acuity - in combination with the red herring technique. The purpose of our experiment was to find out whether people would notice the alternate movie version when they watched a movie via the MORI technique. We disguised the critical questions about noticing the alternate movie version among questions that asked subjects to make social appraisals of the main character in the movie Chad. In short, we lured subjects to "deduce" that we were really interested in their impressions of Chad's personality and behavior.

If subjects reported seeing the alternate movie version, and especially if they did so in response to the least suggestive questions, it would support reviewers' concerns that the MORI technique does not completely block the alternate movie version. In other words, the results from MORI technique research could be explained by people's seeing both versions of a critical detail and later reporting the version that their partner also mentioned in the discussion.

\section{Method}

\section{Subjects}

In total, 40 subjects participated in pairs; 14 were introductory psychology students who took part in return for course credit, and 26 were recruited by advertising around the campus and received a $\$ 10$ grocery voucher.

\section{Design}

We used a between-subjects design. Half of the subjects watched the movie via the standard MORI technique; both versions of the movie were played simultaneously, overlapping on the screen, but one version was blocked with polarization glasses. The remaining subjects watched the movie on the same screen through polarization glasses, but only one version of the movie was played; there was no overlapping image to notice.

\section{Materials and Procedure}

The movie versions were created in the same way as Takarangi, Parker, and Garry's (2006) misinformation materials. Specifically, we filmed a movie and then digitally altered it to create a second version. The movie was about a character named Chad who attended a party. The two versions were identical at all points, except during presentation of the eight critical details (see Table 1 for a list of the critical details). For example, in one version of the movie, Chad stole a wallet, and in the other version, he looked at the wallet but put it back.

The subjects came to the laboratory and sat down at a table facing a rear projection screen. As a cover story, we told the subjects that the study was about people's sensory impressions at different levels of visual acuity. More specifically, at the beginning of the session they were told,

We are interested in people's sensory impressions at different levels of visual acuity. Visual acuity basically means how well you can see. So, right now, you should have $100 \%$ visual acuity, either because your eyes work properly or because you have correcting lenses on. We want to know what happens to people's sensory impressions when their visual acuity is degraded by different amounts.

While explaining the study, the experimenter brought the subjects' attention to a stack of four boxes containing "acuity glasses." Each box was labeled as though it contained different-strength acuity glasses; for example, one box read " $95 \%$ Acuity Glasses: 4 pairs." In fact, only the " $95 \%$ Acuity" box contained glasses; the other boxes were empty but were displayed to add authenticity to the cover story. Next, the subjects were told,

Table 1

Critical Differences Between Movie Versions

\begin{tabular}{lll}
\hline Critical Detail & \multicolumn{1}{c}{ Version 1 } & Version 2 \\
\hline Door & rung bell & knocked \\
Mug & blue & pink \\
Decoration & birthday banner & tinsel \\
Alcohol & vodka & wine \\
Room & laundry & bedroom \\
Balloons & red & blue \\
Drink & squeezed lemon & drugged \\
Put wallet & in his pocket & back on bench \\
\hline
\end{tabular}


Table 2

Subjects' Responses to Each of the Critical Questions

\begin{tabular}{|c|c|c|c|c|}
\hline \multirow[b]{3}{*}{ Critical Question } & \multicolumn{4}{|c|}{ Condition } \\
\hline & \multicolumn{2}{|c|}{$\begin{array}{c}\text { Nonoverlap } \\
\text { (Control) } \\
n=20\end{array}$} & \multicolumn{2}{|c|}{$\begin{array}{c}\text { Overlap (MORI } \\
\text { Technique) } \\
n=20 \\
\end{array}$} \\
\hline & $N$ & $\%$ & $N$ & $\%$ \\
\hline \multicolumn{5}{|l|}{ 1. Anything unusual about movie? } \\
\hline Noticed something unusual & 20 & 100 & 19 & 95 \\
\hline Explanation included double images (or similar)? & 0 & 0 & 1 & 5 \\
\hline \multicolumn{5}{|l|}{ 2. Reasons might disagree with partner } \\
\hline Explanation included seeing different movies (or similar)? & 3 & 15 & 0 & 0 \\
\hline \multicolumn{5}{|l|}{ 3. Red herring (fake purpose for study) } \\
\hline Explanation of true purpose consistent with red herring & 16 & 80 & 17 & 85 \\
\hline Explanation of true purpose included seeing different movies (or similar)? & 0 & 0 & 0 & 0 \\
\hline \multicolumn{5}{|l|}{ 4. Believe saw different movie than did partner? } \\
\hline Said saw different movie from partner & 1 & 5 & 2 & 10 \\
\hline Explanation supported that subject thought saw different movie & 0 & 0 & 0 & 0 \\
\hline
\end{tabular}

Today, you will be in the $95 \%$ acuity condition, so that's not too different to normal vision. I will give you a pair of $95 \%$ acuity glasses, and I am going to show you a movie about a man named Chad, who is at a party; I just want you to watch the movie through the glasses, and then we will do a couple of related tasks afterward. You might find that the movie looks a bit funny through the glasses, but don't worry - that is basically how they are supposed to work. We do find that people see best when they keep their head straight rather than tilted, so we ask that you keep your head as straight and still as possible while you watch the movie. If you already wear glasses, the acuity glasses should fit over the top.

Each subject was given a pair of polarization glasses from the $95 \%$ acuity box. There were two phases to the study: First the subjects watched the movie, and then they completed a question booklet independently.

Phase 1. Each of the movie versions was played on a separate Apple iBook PowerPC G4; each iBook was connected to an NEC ViewLight Mobile DPL projector, Model LT75ZJ, and the images were projected onto the rear of a semitransparent projection screen. The rear projection screen was made from a $1.22 \times 1.82 \mathrm{~m}$ wooden panel and had a $29 \times 38 \mathrm{~cm}$ cutout holding the semitransparent screen. All the equipment was hidden from the subjects' view by the rear projection screen. Two projectors were mounted one above the other in a metal stand, each tilted toward the screen so that the two images overlapped. One movie was polarized horizontally by attaching a polarizing filter horizontally to the projector stand in front of the lens. The other movie was polarized vertically by doing the same with another filter placed at $90^{\circ}$.

Each subject wore either glasses that allowed him or her to see the horizontally polarized image while blocking the vertically polarized image or glasses that did the opposite. The subjects sat at a table facing the rear projection screen for the duration of the study. The movie(s) had no audio and ran for $6 \mathrm{~min} 34 \mathrm{sec}$.

Phase 2. After the pairs watched the movie, each person was given a question booklet to complete independently. The subjects were asked to work through the booklet in order and not to return to any previous pages. The question booklet contained 13 questions. The subjects were asked to provide a written explanation for each answer. Eight of the 13 questions were red herring questions and suggested that we were interested in the subjects' social judgments about Chad. For example, one red herring question read, "If you saw Chad at the party, would you have tried to change his behavior in any way?"

The four critical questions appeared in Positions 5, 10, 11, and 12 and increased in suggestiveness as the subjects progressed through the booklet. The first critical question asked the subjects if they noticed anything unusual about the movie. Next, the subjects listed all the reasons that they could think of why they might not agree with some of their partner's answers if they were asked to discuss the movie together. Then, to test the red herring, the third question asked the subjects what they thought the true purpose of the study was. The last critical question asked the subjects whether they thought that they had seen the same movie as the person next to them. Finally, Question 13 asked the subjects what they knew about the study before they took part.

\section{Results and Discussion}

The goal of this experiment was to determine whether subjects would notice an alternate movie version when watching a movie via the MORI technique. To that end, we examined the subjects' responses to each of the four critical questions. One coder rated the responses given by all the subjects, and a second independent coder rated the responses given by a random $25 \%$ of the sample. Interrater agreement was at $100 \%$ in every category. Below, we will address each of the four critical questions in turn (see Table 2 for a summary of the subjects' responses to each of the critical questions).

Did the subjects notice anything unusual about the movie? As is shown in Table 2, every subject in the nonoverlap group and the majority of subjects in the overlap group reported noticing something unusual about the movie. However, except for one, the subjects' explanations about what was unusual were not relevant to our experiment (e.g., some subjects reported that the lack of audio was unusual).

Only 1 subject (from the overlap group) reported seeing multiple images in response to this question; she wrote, "Chad's actions kind of doubled like when you have bad reception on TV and you see 2 people rather than one." Crucially, this subject did not report seeing any conflicting details, merely poor-quality images. In addition, she did not conclude that the double images resulted from different movies; instead, she later reported that she thought that she had seen the same movie as her partner and gave the explanation, "There's one screen. How can there be two movies playing?" 
Reasons for disagreement. Next, the subjects listed all the reasons that they might not agree with their discussion partner when talking about the movie. As is shown in Table 2, only 3 subjects mentioned the possibility that they might have seen something different from their partners. All 3 were in the nonoverlap group and also listed other possibilities, suggesting that they were simply noting a number of hypothetical scenarios, instead of genuinely believing that they had seen something different.

True purpose of the study. As is shown in Table 2, the red herring was effective at hinting at a fake reason for the study: The majority of the subjects' responses were consistent with the red herring theme and were focused on judgments of behavior. Not 1 subject mentioned anything to do with multiple images in response to this question.

Did subjects think they had seen a different movie from their partner? Table 2 shows that most subjects thought that they had seen the same movie as their partner. In fact, many subjects expressed surprise at the question. For example, the subjects' explanations included:

Subject Pa: There was only one screen and we were both looking at it together, I can't imagine how we both could have watched different movies simultaneously on the same screen ....

Subject Rb: We were in the same room at the same time watching the same movie-I don't think the glasses were going to change that.

However, as Table 2 shows, 3 subjects were not sure that they had seen the same movie as their partner. One of these subjects was in the nonoverlap condition and could not have seen the alternate movie. He deduced that there were two movies on the basis of our questions.

Subject Da: Because the questions before that on "if you two were asked about the movie do you think you may disagree on things?" seemed to imply that we would disagree on things because we did in fact watch different movies.

The other 2 subjects were in the overlap group. Rather than believing there were two separate movies, 1 of these subjects suggested that people would experience the same movie differently depending on their own histories.

\section{Subject Ga: We both would have assumed Chad to be doing different things depending on our past experiences.}

Finally, the other subject gave an ambiguous response, and his explanation provided no evidence that he had noticed the alternate movie version.

Subject Fa: I sort of thought maybe we were seeing different movies - we laughed at different times. Might just be because we thought those bits were funny, but I don't really know.
In summary, there is, at best, only weak evidence that subjects might notice the alternate movie version when they watch a movie via the MORI technique. The subjects were not suspicious that we might have shown them different movies; despite the demand of the critical questions, the 1 subject who noticed overlapping images did not think the different images came from different movies. The results suggest that the MORI technique is an effective way to show different people different movies on the same screen without realizing it. At worst, on the rare occasions that people notice double images, it is unlikely that they will conclude that there are two movies. However, there is one other way that the MORI technique could give the illusion that it works when it does not. If the subjects had seen conflicting details without realizing it, they would have been unable to answer the explicit questions we asked in Experiment 1, but their implicit memory of the alternate movie could have influenced their responses on a memory test. For instance, perhaps a subject saw both the laundry and the bedroom critical detail and was simply more likely to report the laundry detail (from the alternate version) when his or her partner also mentioned seeing the laundry. In Experiment 2, we examined the possibility that subjects' implicit memories of the alternate movie drive the effects behind the MORI technique.

\section{EXPERIMENT 2}

To investigate whether subjects would have implicit memories of the alternate movie version, we used a second-choice memory test (see E. F. Loftus, 1996). For each question, the subjects gave two answers: the answer that they thought was most accurate (their first choice) and the answer that they thought was second most likely to be accurate (their second choice). Because the subjects were not restricted to providing only one response, if they saw both versions of a critical detail, they would be able to report both on the test. Of course, one caveat is that subjects might also come to report the alternative version of a critical detail not because they remembered it, but because it seemed the most likely possibility of the alternatives listed. For instance, if someone saw Chad knock on the door, they might think that the next most likely action would be that that he would ring the bell. In short, they might pick "rung the bell" as the second choice, even though they never saw it. To control for this possibility, we compared responses for overlapped details (details presented using the standard MORI technique) with responses for nonoverlapped details (details for which the subjects could not have any memory of the alternate movie). In other words, we compared how often people reported a critical detail when it appeared in the alternate version (overlapped) and how often they picked the same detail by chance alone when it was not in the alternate version and there was absolutely no possibility of seeing it (nonoverlapped). If subjects were more likely to report the critical details from the alternate movie version for overlapped details, it would provide evidence that they knew 
something about the alternate version, whether or not they were aware of it (E. F. Loftus, 1996; Wright, 1993).

If subjects have implicit memories for aspects of the alternate movie, their responses might also be affected in other ways. For instance, the overlapped image might somehow affect subjects' ability to remember the details that they did see in their own version of the movie; perhaps the MORI technique inherently harms people's memory accuracy. The design of our experiment also allowed us to address this additional question. Specifically, if the subjects' ability to remember the details from their own version of the movie was hurt by the MORI technique, they would be less likely to report critical details from their own version of the movie when the details were overlapped than when the details were nonoverlapped.

In sum, if the subjects were poorer at remembering overlapped details, less confident when remembering overlapped details, or more likely to choose the answer from the alternate movie version for overlapped details, it would provide evidence that people's memories are influenced by the alternate version when movies are presented via the MORI technique.

\section{Method}

\section{Subjects}

In total, 40 introductory psychology students took part in return for course credit. They participated individually, in sessions with no more than 2 subjects.

\section{Design}

Just as with our previous MORI technique research (French et al., 2008; Garry et al., 2008), we used a within-subjects design; instead of comparing subjects' memories for discussed and nondiscussed details, we compared subjects' memories for overlapped and nonoverlapped details. The subjects saw one version of the Chad movie with half of the critical details overlapped with the alternate movie version, using the standard MORI technique, and half of the details not overlapped with any image.

\section{Materials and Procedure}

Movie. We followed the same method as that in Experiment 1, except that we used four new versions of the Chad movie in combination with the original two versions. To create the four new versions, we split each of the original versions into two new half-length movies; each new half-version included four of the eight critical details (see Table 1). We used iMovie to fill up the remaining duration of the new movies with a blank image; the half-length movies kept time with the full-length movies, but nothing was projected onto the screen for the blank half. In the first-half movie versions, the Chad movie played for $3 \mathrm{~min} 3 \mathrm{sec}$ and then faded into a blank image for the remaining $3 \mathrm{~min} 31 \mathrm{sec}$; for the second-half movies, a blank image played for $3 \mathrm{~min} 3 \mathrm{sec}$ and then faded into the Chad movie, which played for the remaining $3 \mathrm{~min} 31 \mathrm{sec}$.

For example, a subject might have been shown Version 1 of the movie overlapped with the new Version 2 first-half movie. In this case, the first four critical details in the movie would have acted as overlapped details. After these details were presented ( $3 \mathrm{~min} 3 \mathrm{sec}$ through the movie) the Version 2 first-half movie faded to a blank image, and the remaining four critical details would have acted as nonoverlapped details. Critical details were fully counterbalanced and acted equally often as overlapped and nonoverlapped details.

In order to parallel the time the subjects in Garry et al. (2008) and French et al. (2008) took to complete the filler tasks and discussion (between the movie and the recognition test), after the movie, the

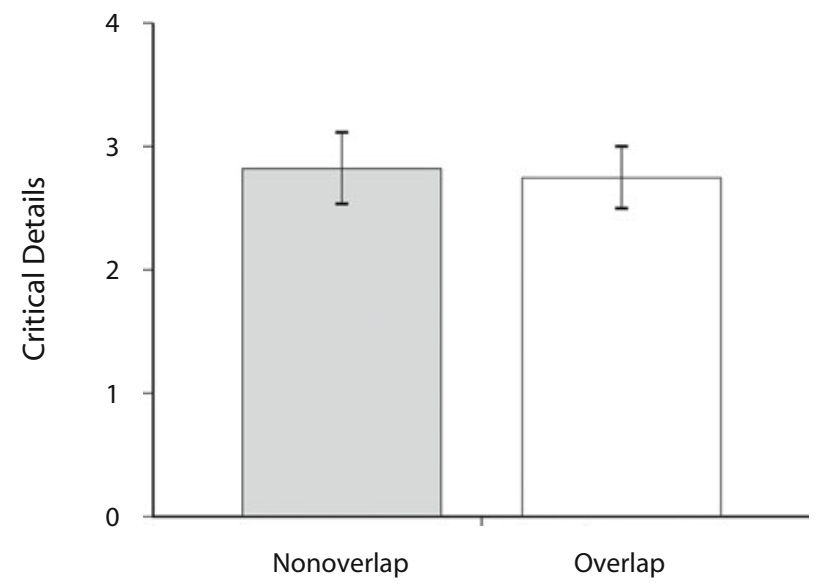

Figure 2. Number of times subjects reported critical details from their own movie version for nonoverlapped and overlapped details. Error bars depict $95 \%$ confidence intervals of the means.

subjects completed filler tasks for $20 \mathrm{~min}$. After the filler tasks, the subjects completed a second-choice recognition test.

Test. There were 12 questions on the test; 8 were related to the eight critical details, and 4 were fillers. Each question asked about a detail from the movie and suggested five answers. For critical questions, the correct answer from each movie version was listed among the five alternatives. For example, one question read, "Where did Chad put the wallet that he found on the bench? In the cupboard; Behind the microwave; Back where he found it; In his back pocket; In his bag." The subjects selected their answer and rated their confidence on a 5 -point scale, where $1=$ not at all confident and $5=$ very confident. The subjects were then asked to answer the same question again, providing their next best response. Finally, the subjects were asked to explain why they had selected their second response.

\section{Results and Discussion}

Recall that implicit memories of the alternate movie might affect people's responses in a number of different ways. First, people's ability to remember the critical details from their own version of the movie might be hurt. To address this question, we calculated the number of times the subjects reported critical details from their own version of the movie when the details were overlapped and nonoverlapped. Put another way, we compared how well the subjects remembered the critical details from their own version of the movie for the overlapping and nonoverlapping segments of the movie; we display these data and the corresponding confidence intervals in Figure 2. As the figure shows, the subjects' initial responses were just as likely to be correct whether the details were overlapped or not $(t<1$, n.s.), and the confidence intervals around the means were narrow. In addition, the confidence interval for the difference between the means was narrow and included zero, suggesting that the null results were not due to a lack of power $(95 \% \mathrm{CI}=-0.49<\mu 1-\mu 2<$ 0.33; Aberson, 2002; G. R. Loftus, 1996). Finally, the effect size and corresponding confidence interval were also small, providing more support for the null result (Cohen's $d=.06,95 \% \mathrm{CI}=-0.34<D<0.21$; Aberson, 2002; Thompson, 2002). ${ }^{1}$ Taken together, these results suggest 
that presenting a movie via the MORI technique did not affect the subjects' memories of the critical details from their own version of the movie or their ability to report those memories.

Even though the overlapping movie did not influence the subjects' memories, it might have affected the subjects' subjective experiences of their memories. To address this possibility, we next examined the subjects' confidence in their responses. As Figure 3 shows, the subjects were just as confident in their answers for overlapped and nonoverlapped details $(t<1, \mathrm{n}$.s.). The confidence intervals around the means were narrow, and the confidence interval for the difference between the means was small $(95 \%$ $\mathrm{CI}=-0.28<\mu 1-\mu 2<0.35)$, as was the corresponding effect size (Cohen's $d=.05,95 \% \mathrm{CI}=-0.18<D<$ 0.25 ), suggesting that these null results were not due to a lack of power.

Considered together, these results lend support to the idea that the alternate movie version did not affect people's ability to remember details from their own version of the movie or their subjective appraisals of their memories. People were just as good at remembering overlapped and nonoverlapped details and just as confident at remembering overlapped and nonoverlapped details.

Our next research question was whether the subjects had any memory for the details in the alternate movie version. To answer this question, we first calculated the number of times the subjects reported critical details from the alternate movie version; we compared subjects' responses for overlapped and nonoverlapped details across their first and second responses. As Figure 4 shows, the subjects did not demonstrate any memory of the critical details from the alternate movie version; they were equally likely to report critical details whether the details were overlapped or nonoverlapped for both their first responses and their second responses (both $t \mathrm{~s}<1$, n.s.), and, consistent with the other analyses, the confidence intervals around the means were narrow. In addition, the confidence intervals for the differences between means were also narrow and included zero, suggesting that the results were not due to a lack of power $(95 \% \mathrm{CI}=-0.34<\mu 1-\mu 2<0.29$ and $95 \% \mathrm{CI}=-0.54<\mu 1-\mu 2<0.24$, respectively). The effect size of the difference for the subjects' first choices was small (Cohen's $d=.03,95 \% \mathrm{CI}=-0.24<D<$ 0.18 ), further supporting the null result. However, there was a more substantial effect size for the subjects' second choices (Cohen's $d=.12,95 \% \mathrm{CI}=-0.07<D<0.45$ ). More important is what Figure 4 shows: If anything, the subjects were slightly more likely to report critical details from the alternate version when they were nonoverlapped, a tendency opposite to that expressed by reviewers. Taken together, these results suggest that the subjects had no memory for the alternate movie version.

Finally, if subjects remembered both versions of a critical detail and they were aware of it, they should say so when explaining their second response. To address this issue, we examined the subjects' explanations for why they chose their second answers. Consistent with the results of Experiment 1, there was not a single instance in which a

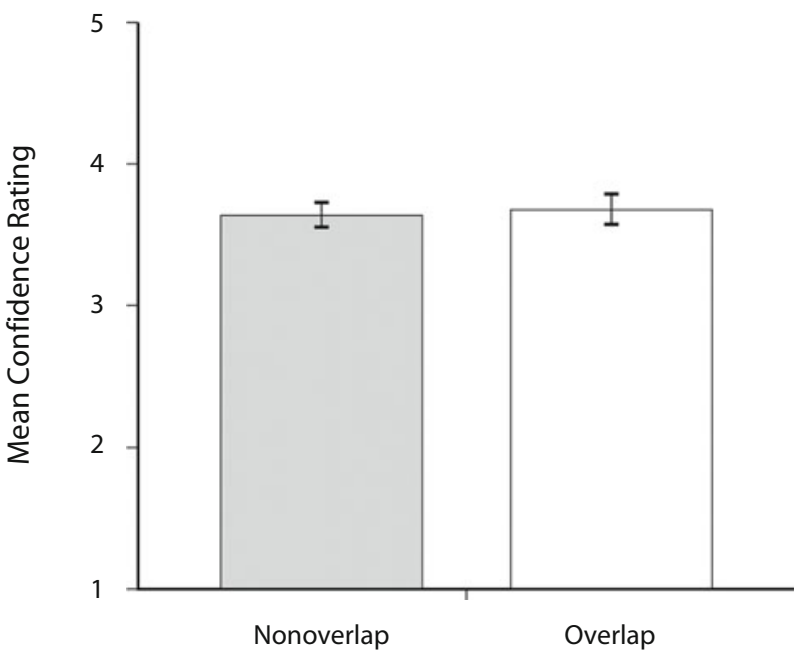

Figure 3. Mean confidence for nonoverlapped and overlapped details. Error bars depict $95 \%$ confidence intervals of the means.

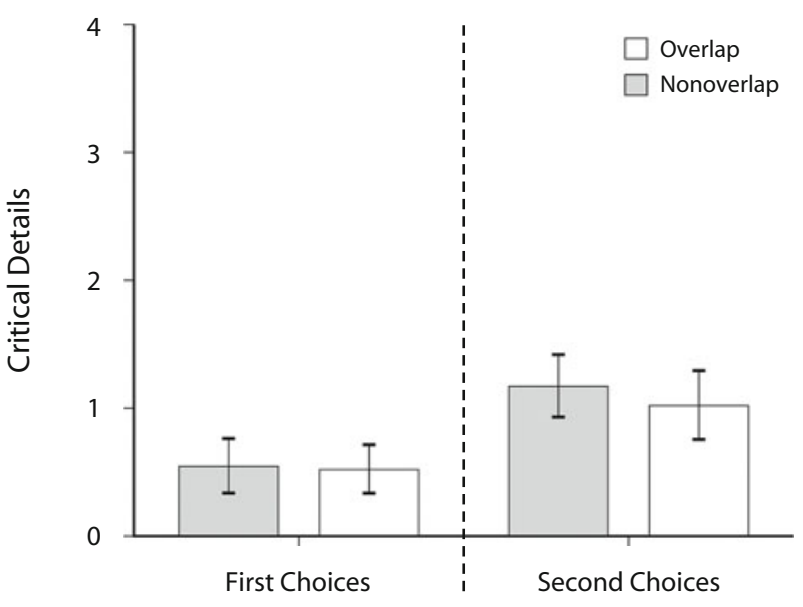

Figure 4. Number of times subjects reported critical details from the alternate movie in their first and second choices for nonoverlapped and overlapped details. Error bars depict $\mathbf{9 5 \%}$ confidence intervals of the means.

subject mentioned anything even vaguely related to seeing both versions of a detail. By far the most common way that the subjects selected their second choice was through logical reasoning; for $71 \%$ of the explanations given, the subjects selected their second choice by reasoning that it made sense or by excluding other alternatives. Other strategies that the subjects used to make their second choices included guessing, beliefs about base rates, personal preferences, and expectations.

\section{GENERAL DISCUSSION}

Research using the MORI technique has shown that people will report seeing details that they did not see, details that another person merely described to them (French et al., 2008, 2009; Garry et al., 2008). But is this effect merely a methodological flaw masquerading 
as a memory distortion? The answer is no. In two experiments, we found no evidence that the MORI technique leads people to see both versions of an event. In Experiment 1, the subjects were generally not suspicious that they might have seen different movies, and all but 1 failed to notice any overlapping images. In addition, the 1 subject who did notice overlapping images did not think that the different images came from different movies. In Experiment 2, there was no evidence that the MORI technique harmed the subjects' ability to remember their own versions of the critical details. Subjects were just as good at remembering overlapped and nonoverlapped details, just as confident when remembering overlapped and nonoverlapped details, and no more likely to report the critical details from the alternate movie version for overlapped or nonoverlapped details. Moreover, their explanations did not include noticing the alternate movie version. In short, we found no evidence for these MORI technique counterexplanations. Instead, we found evidence that the MORI technique is a sound tool, one that allows researchers to create and examine social situations that would not be possible otherwisea tool that complements the tools used in other research (e.g., Cuc et al., 2006; Gabbert et al., 2004; Paterson \& Kemp, 2006; Roediger et al., 2001; Wright et al., 2005) and will help to give us a more complete understanding of social influences on memory than we would gain using one approach alone.

\section{AUTHOR NOTE}

We are grateful for the support of the New Zealand Government through the Marsden Fund, administered by the Royal Society of New Zealand on behalf of the Marsden Fund Council. This research was supported by a Grant-in-Aid from the Japanese Ministry of Education, Culture, Sports, Science, and Technology (Grant 16330139) to K.M. Our thanks to Thomas Huthwaite for his help with coding. Correspondence concerning this article should be addressed to M. Garry, School of Psychology, Victoria University of Wellington, P.O. Box 600, Wellington, New Zealand (e-mail: maryanne.garry@vuw.ac.nz).

\section{REFERENCES}

ABERSON, C. (2002). Interpreting null results: Improving presentation and conclusions with confidence intervals. Journal of Articles in Support of the Null Hypothesis, 1, 36-42.

Cuc, A., Ozuru, Y., Manier, D., \& Hirst, W. (2006). On the formation of collective memories: The role of a dominant narrator. Memory \& Cognition, 34, 752-762.

DodD, D. H., \& Bradshaw, J. M. (1980). Leading questions and memory: Pragmatic constraints. Journal of Verbal Learning \& Verbal Behavior, 19, 695-704. doi:10.1016/S0022-5371(80)90379-5

Echterhoff, G., Hirst, W., \& Hussy, W. (2005). How eyewitnesses resist misinformation: Social postwarnings and the monitoring of memory characteristics. Memory \& Cognition, 33, 770-782.

French, L., GarRY, M., \& Mori, K. (2008). You say tomato? Collaborative remembering between intimate couples leads to more false memories than collaborative remembering between strangers. Memory, 16, 262-273. doi:10.1080/09658210701801491

French, L., Garry, M., \& Mori, K. (2009). Social factors determine the influence of discussion on memory. Manuscript under review.

French, L., Sutherland, R., \& Garry, M. (2006). Discussion affects memory for true and false childhood events. Applied Cognitive Psychology, 20, 671-680. doi:10.1002/acp.1219

Gabbert, F., Memon, A., Allan, K., \& Wright, D. B. (2004). Say it to my face: Examining the effects of socially encountered mis- information. Legal \& Criminological Psychology, 9, 215-227. doi:10.1348/1355325041719428

Gabbert, F., Memon, A., \& Wright, D. B. (2006). Memory conformity: Disentangling the steps toward influence during a discussion. Psychonomic Bulletin \& Review, 13, 480-485.

Gabbert, F., Memon, A., \& Wright, D. B. (2007). I saw it for longer than you: The relationship between perceived encoding duration and memory conformity. Acta Psychologica, 124, 319-331. doi:10.1016/j .actpsy.2006.03.009

Garry, M., French, L., Kinzett, T., \& Mori, K. (2008). Eyewitness memory following discussion: Using the MORI technique with a Western sample. Applied Cognitive Psychology, 22, 431-439. doi:10.1002/acp. 1376

Hirokawa, K., Matsuno, E., Mori, K., \& Ukita, J. (2006). Relationship between masculinity-femininity and concession in an experimental collaborative eyewitness testimony. Asian Journal of Social Psychology, 9, 132-139. doi:10.1111/j.1467-839X.2006.00190.x

Ikier, S., TekCan, A. I., Gulgoz, S., \& Kuntay, A. C. (2003). Whose life is it anyway? Adoption of each other's autobiographical memories by twins. Applied Cognitive Psychology, 17, 237-247. doi:10.1002/ acp. 869

Johnson, M. K., Hashtroudi, S., \& Lindsay, D. S. (1993). Source monitoring. Psychological Bulletin, 114, 3-28. doi:10.1037/0033 $-2909.114 .1 .3$

Kanematsu, H., Mori, K., \& Mori, H. (2003). Memory distortion in eyewitness pairs who observed nonconforming events and discussed them. Journal of Faculty of Education, Shinshu University, 109, 75-84

Kassin, S. M., \& Kiechel, K. L. (1996). The social psychology of false confessions: Compliance, internalization, and confabulation. Psychological Science, 7, 125-128. doi:10.1111/j.1467-9280.1996 .tb00344.x

Laney, C., KaAsa, S. O., Morris, E. K., Berkowitz, S. R., Bernstein, D. M., \& LoftUS, E. F. (2008). The red herring technique: A methodological response to the problem of demand characteristics. Psychological Research, 72, 362-375. doi:10.1007/s00426-007-0122-6

LinDSAY, D. S. (2008). Source monitoring. In J. Byrne (Series Ed.) \& H. L. Roediger III (Vol. Ed.). Learning and memory: A comprehensive reference. Vol. 2: Cognitive psychology of memory (pp. 325-348). Oxford: Elsevier.

Loftus, E. F. (1996). Eyewitness testimony. Cambridge, MA: Harvard University Press.

Loftus, E. F., Levidow, B., \& Duensing, S. (1992). Who remembers best? Individual differences in memory for events that occurred in a science museum. Applied Cognitive Psychology, 6, 93-107. doi:10.1002/acp.2350060202

Loftus, G. R. (1996). Psychology will be a much better science when we change the way we analyze data. Current Directions in Psychological Science, 5, 161-171. doi:10.1111/1467-8721.ep11512376

MeAde, M. L., \& Roediger, H. L., III (2002). Explorations in the social contagion of memory. Memory \& Cognition, 30, 995-1009.

Mitchell, K. J., \& Johnson, M. K. (2000). Source monitoring: Attributing mental experiences. In E. Tulving \& F. I. M. Craik (Eds.), The Oxford handbook of memory (pp. 179-195). New York: Oxford University Press.

MoRI, K. (2003). Surreptitiously projecting different movies to two subsets of viewers. Behavior Research Methods, Instruments, \& Computers, 35, 599-604.

MorI, K. (2007). A revised method for projecting two different movies to two groups of viewers without their noticing the duality. Behavior Research Methods, 39, 574-578.

ORNE, M. T. (1962). On the social psychology of the psychological experiment with particular reference to demand characteristics and their implications. American Psychologist, 17, 776-783. doi:10.1037/ h0043424

Paterson, H. M., \& Kemp, R. I. (2006). Comparing methods of encountering postevent information: The power of co-witness suggestion. Applied Cognitive Psychology, 20, 1083-1099. doi:10.1002/acp.1261

Roediger, H. L., III, Meade, M. L., \& Bergman, E. T. (2001). Social contagion of memory. Psychonomic Bulletin \& Review, 8, 365-371.

SCHNEIDER, D. M., \& WATKINS, M. J. (1996). Response conformity in recognition testing. Psychonomic Bulletin \& Review, 3, 481-485. 
ShaW, J. S., III, Garven, S., \& Wood, J. M. (1997). Co-witness information can have immediate effects on eyewitness memory reports. Law \& Human Behavior, 21, 503-523. doi:10.1023/A:1024875723399

Sheen, M., Kemp, S., \& Rubin, D. (2001). Twins dispute memory ownership: A new false memory phenomenon. Memory \& Cognition, 29, 779-788.

Takarangi, M. K. T., Parker, S. L., \& Garry, M. (2006). Modernising the misinformation effect: The development of a new stimulus set. Applied Cognitive Psychology, 20, 1-8. doi: 10.1002/acp.1209

Thompson, B. (2002). What future quantitative social science research could look like: Confidence intervals for effect sizes. Educational Researcher, 31, 25-32. doi:10.3102/0013189X031003025

Tousignant, J. P., Hall, D., \& Loftus, E. F. (1986). Discrepancy detection and vulnerability to misleading postevent information. Memory \& Cognition, 14, 329-338.

WRIGHT, D. B. (1993). Misinformation and warnings in eyewitness testimony: A new testing procedure to differentiate explanations. Memory, 2, 153-166. doi:10.1080/09658219308258229
Wright, D. B., Mathews, S. A., \& Skagerberg, E. M. (2005). Social recognition memory: The effect of other people's responses for previously seen and unseen items. Journal of Experimental Psychology: Applied, 11, 200-209. doi:10.1037/1076-898X.11.3.200

Wright, D. B., Self, G., \& Justice, C. (2000). Memory conformity: Exploring misinformation effects when presented by another person. British Journal of Psychology, 91, 189-202. doi:10.1348/000712600161781

\section{NOTE}

1. Effect sizes were calculated using $G^{*}$ Power Version 3.0.10. Confidence intervals for the effect sizes were obtained using the calculator available at http://wilderdom.com/301/tutorials/Tutorial7-Power.html.

(Manuscript received January 31, 2009; revision accepted for publication May 20, 2009.) 